\title{
Object-substitution masking degrades the quality of conscious object representations
}

\author{
Geoffrey W. Harrison ${ }^{1}$. Jason Rajsic ${ }^{2}$ • Daryl E. Wilson ${ }^{1}$
}

Published online: 17 June 2015

(C) Psychonomic Society, Inc. 2015

\begin{abstract}
Object-substitution masking (OSM) is a unique paradigm for the examination of object updating processes. However, existing models of OSM are underspecified with respect to the impact of object updating on the quality of target representations. Using two paradigms of OSM combined with a mixture model analysis we examine the impact of postperceptual processes on a target's representational quality within conscious awareness. We conclude that object updating processes responsible for OSM cause degradation in the precision of object representations. These findings contribute to a growing body of research advocating for the application of mixture model analysis to the study of how cognitive processes impact the quality (i.e., precision) of object representations.
\end{abstract}

Keywords Object-substitution masking • Object-updating · Consciousness

\section{Introduction}

A fundamental goal in the study of perception is the delineation of the processes that transform sensory information into objects of consciousness (Dehaene \& Naccache, 2001). To that end, visual masking has been an influential tool in probing the boundaries between conscious and unconscious vision (Breitmeyer, 2007). Typically, masking refers to the disruption

Geoffrey W. Harrison

8gh3@queensu.ca

1 Department of Psychology, Queen's University, Kingston, ON K7L 3N6, Canada

2 Department of Psychology, University of Toronto, Toronto, ON, Canada in processing of a target object by a temporally proximate second object (the mask) that either overlaps or shares a contiguous spatial boundary with the target (Breitmeyer \& Öğmen, 2006). Due to the spatial contiguity between target and mask, these standard masking procedures disrupt low level or early visual processes by merging the target and mask into a single indecipherable percept. This feature makes standard masking procedures unsuitable for addressing the later perceptual processing mechanisms that underlie conscious awareness.

Unlike standard masking procedures, object substitution masking (OSM), has properties that make it ideal for studying the later perceptual processing stages contributing to conscious awareness (Di Lollo, Enns, \& Rensink, 2000; Goodhew, Pratt, Dux, \& Ferber, 2013). Typically, an OSM paradigm involves the brief presentation of one or more objects with one of the objects (the target) surrounded by four small dots (the mask). On simultaneous mask trials, the target (and all other objects) and mask onset and offset together. On delayed mask trials, they onset together but the mask offset is delayed relative to the target and other objects. Effective masking is evidenced by reduced accuracy in reporting either the presence of, or some characteristic of, the target object in the delayed mask condition. Unlike other forms of masking whose results are explained primarily by disruptions to object formation mechanisms (see Enns, 2004, for a review), several unique characteristics of OSM (e.g., common onset of the target and mask, sparseness of the mask) support the notion that its effects result from conflict between object-based representations (Di Lollo et al., 2000; Lleras \& Moore, 2003; Moore \& Lleras, 2005; but see Põder, 2013). This object substitution account of masking describes a communicative process that resolves discrepancies between late and early stages of visual processing in an attempt to determine the appropriate conscious representation. In the case of OSM, this results in 
the target's representation being updated with that of the mask, and the latter reflecting conscious experience.

To date, studies examining OSM have used only coarse measures of target awareness (e.g., present versus absent; oriented left versus right), an approach that has resulted in theories of OSM being underspecified with respect to the impact of OSM on the quality of target representations. In fact, object-mediated accounts of OSM contend that either the target representation is eliminated before a conscious representation is produced (e.g., Di Lollo et al., 2000) or a conscious target representation is overwritten by the mask's representation (e.g., Lleras \& Moore, 2003). An outstanding question then remains regarding whether OSM results in a complete loss of target information, or whether there is a partial loss such that the target is degraded but still accessible to consciousness.

In the working memory (WM) literature, a novel method for measuring the quality of representation of object properties has gained traction recently (Bays, Catalao, \& Husain, 2009; Zhang \& Luck, 2008). This method requires participants to retrieve a particular feature of a memory item and to respond using a continuous scale. On a typical trial, participants might be instructed to remember the orientation of several memory items and, after a short delay, reproduce the orientation of one item. This means that, unlike standard WM tasks, responses are not dichotomized as correct or incorrect. By fitting a mixture model, one can estimate the proportion of trials where responses came from a circular Gaussian distribution centred on target's orientation, guess trials where responses came from a uniform distribution, and non-target trials in which responses came from a circular Gaussian centered on a different item. Critically, the standard deviation for the target responses' distribution provides a quantifiable measure of the quality (i.e., precision) of the memory target feature.

To our knowledge, the mixture modelling approach has been applied to conscious perception in just one study. Asplund and colleagues (2014) showed that, during an attentional blink, discrete representations are lost, but the precision of object representations is unaffected. This led to the conclusion that conscious perception may be all-or-none. However, the attentional blink and OSM may rely on distinct mechanisms of conscious perception (Giesbrecht, Bischof, \& Kingstone, 2003). The goal of this paper then was to adapt the mixture model analysis to the OSM paradigm and, by doing so, elucidate the impact of OSM on the quality of target representations. If OSM eliminates conscious object representations, as reported for the attentional blink, then the delayed mask should have no effect on target precision, but reduce the proportion of target responses and an increase the proportion of guess responses. If, however, OSM operates by degrading conscious representations, then there should be a reduction in target precision.

\section{Experiment 1}

\section{Method}

\section{Participants}

Twenty-three undergraduate students from Queen's University participated in exchange for credit in an introductory psychology course. All had normal or corrected-to-normal vision, and were naïve to the experiment's purpose.

\section{Apparatus}

The experiment was conducted on a personal computer in a dimly lit room. Stimuli were presented with Psychophysics Toolbox version 3.0.8 (Brainard, 1997) in MATLAB version 7.04 on a 16 -inch CRT monitor. A chin-rest kept viewing distance constant at $50 \mathrm{~cm}$. Responding was done with a Logitech t650 touchpad and keyboard.

\section{Design and procedure}

There were six blocks of 144 trials for a total of 864 trials. See Fig. 1 for a typical trial sequence. Following a 500-ms fixation display, the initial display (17 ms duration) consisted of 2, 4 or 8 circular Landolt-Cs (radius of $0.5^{\circ}$ ) presented on a dark-gray background distributed equally on an imaginary circle with a radius of $3.5^{\circ}$ from a central fixation cross (subtending $1.0^{\circ}$ ). The gap in each Landolt-C had an independent random orientation $\left(0^{\circ}-359^{\circ}\right)$ and was approximately one-quarter the circumference of a completed circle. On each trial, one Landolt$\mathrm{C}$ (the target) was selected randomly to be surrounded by four small white dots (each subtending $0.1^{\circ}$ ) forming the corners of a square $\left(0.6^{\circ}\right.$ by $\left.0.6^{\circ}\right)$. The four dot mask either offset with the initial search array (simultaneous mask condition) or remained for an additional 150 or 300 ms (delayed mask conditions). Participants were instructed to produce a quick, accurate judgment of the target's gap orientation by swiping their index finger along the touchpad as if they were passing it through the midpoint of the target's gap from the center of the Landolt-C. ${ }^{1}$ During responding, a Landolt-C was drawn at the target location with a gap angle that matched the finger's movement trajectory allowing for fine-tuning of responses. Set size and mask conditions were randomized with an equal number of trials in each condition.

\footnotetext{
${ }^{1}$ We also collected pilot data where participants used a mouse to produce their gap orientation response and clicked the left mouse button to confirm their response. Overall data patterns and statistical results did not differ across the two approaches but response variance estimates across conditions were more reliable and the total experiment time was reduced by approximately $15 \%$ by using the touchpad.
} 


\section{Data analysis}

For each trial, orientation error was the difference between the target's actual and reported orientation in degrees. Orientation errors for each of the nine conditions (three set sizes and three mask durations) were fitted to the three-component model of Bays et al. (2009). This model assumes a weighted mixture of three response types: (1) target responses, defined as correctly reporting the target orientation; (2) guess responses, defined as randomly guessing an orientation value; and (3) non-target responses, defined as reporting the orientation of a nontarget object. Target and non-target responses were modelled with circular Gaussian distributions termed von Mises distributions, whereas guess responses were modeled with a uniform distribution. The concentration parameter (measure of spread) of each von Mises distribution was fit to the same parameter and then converted to a standard deviation using a mathematical transformation described by Bays et al. (2009). Thus, four parameter estimates were derived for each condition for each participant: (1) target responses ( $\left.\mathrm{P}_{\text {Target }}\right)-$ proportion of trials in which there was memory of the target's orientation, (2) non-target responses $\left(\mathrm{P}_{\text {Non-target }}\right)$ - proportion of trials in which a non-target's orientation was reported, (3) guess responses $\left(\mathrm{P}_{\text {Guess }}\right)$ - proportion of trials in which there was no memory and orientation was guessed, and (4) gap variance (SD) — standard deviation of the circular Gaussian distribution component for the non-guess responses. ${ }^{2}$ Note that SD is inversely related to precision.

\section{Results and discussion}

Data from five participants was excluded due to poor performance $\left(\mathrm{P}_{\text {Target }}\right.$ in simultaneous mask offset conditions $<50 \%$ ). Four 3 (set size) $\times 3$ (mask duration) repeated measures analyses of variance (ANOVAs) were conducted - one for each of the derived parameter estimates $\left(\mathrm{P}_{\text {Target }}, \mathrm{P}_{\text {Non-target }}, \mathrm{P}_{\text {Guess }}\right.$, and $\mathrm{SD}$ ) (see Fig. 2). The ANOVAs for both $\mathrm{P}_{\text {Target }}$ and $\mathrm{P}_{\text {Guess }}$ showed main effects of set size and mask duration, and an interaction, all $F$ s $>4.61$, all $P$ values $<.002$, all partial $\eta^{2}>.21$. To investigate the interaction, OSM magnitude for each set size was calculated

\footnotetext{
${ }^{2}$ We thank an anonymous reviewer for raising the possibility that an OSM paradigm may be better described with a model that does not assume equal precision for target and non-target (distractor) responses. When including a fifth parameter for the precision of non-target responses $\left(\mathrm{SD}_{\text {Non-target }}\right)$ in the model, the impact of set size and mask duration on the other four estimates $\left(\mathrm{P}_{\text {Target }}, \mathrm{P}_{\text {Non-target, }}, \mathrm{P}_{\text {Guess }}, \mathrm{SD}_{\text {Target }}\right)$ was the same as observed in the original four parameter model. Furthermore, no effects of set size and mask duration were observed on $\mathrm{SD}_{\text {Non-target. }}$ Because $\mathrm{P}_{\text {Non-target }}$ was near zero for many participants, making estimating non-target precision problematic (typically, a minimum of 50 trials is required for reliable precision estimates), and because application of the five parameter model, with separate parameters to estimate the precision of target and non-target responses, did not alter any of the effects in our results, we opted to focus on the four-parameter model.
}

as the difference between simultaneous and the average of the delayed mask conditions. OSM magnitude for $\mathrm{P}_{\text {Target }}$ increased from set size $2(8.5 \%)$ to $4(16.6 \%)$ and from set size 4 to 8 (25.8\%), all $t \mathrm{~s}>2.88$, all $P_{\mathrm{s}}<.01$. For $\mathrm{P}_{\text {Guess }}$, OSM magnitude did not differ between set sizes $2(6.9 \%)$ and $4(11.3 \%), t(17)=$ $1.51, P=.15$, but increased from set size 4 to $8(20.4 \%), t(17)=$ 3.12, $P=.006$.

For $\mathrm{P}_{\text {Non-target }}$ there was a main effect of mask duration, $F(2,34)=37.65, P<.001$, partial $\eta^{2}=.69$; however, there was no main effect of set size and no interaction, all $F_{\mathrm{S}}<2.16$, all $P_{\mathrm{s}}>.13$, all partial $\eta^{2} \mathrm{~s}<.11$. Contrast analyses revealed that $\mathrm{P}_{\text {Non-target }}$ increased linearly across mask durations, $P=.003$, partial $\eta^{2} \mathrm{~s}=.42$.

For SD (see Fig. 2b), there was a main effect of set size, $F(2,34)=23.08, P<.001$, partial $\eta^{2}=.58$, and mask duration, $F(2,34)=25.93, P<.001$, partial $\eta^{2}=.60$; however, the interaction was not significant, $F(1,280.07)=2.69, P=.12$, partial $\eta^{2}=.14$. Contrast analyses revealed a linear increase in variability (i.e., decrease in precision) across set sizes and mask durations, all $P_{\mathrm{s}}<.001$, all partial $\eta^{2}>$.68. However, post-hoc tests showed that there were no differences in SD between 150 and $300 \mathrm{~ms}$ delayed mask durations for any set sizes, all $t \mathrm{~s}<.96$, all $P \mathrm{~s}>.35$.

Importantly, our $\mathrm{P}_{\text {Target }}$ estimates mirror those from the OSM literature using either forced choice or presence/ absence discrimination procedures (e.g., Di Lollo et al., 2000) in showing both decreases in $P_{\text {Target }}$ with increasing set size, and reduced $\mathrm{P}_{\text {Target }}$ for delayed relative to simultaneous offset mask conditions (and vice-versa for $\mathrm{P}_{\mathrm{Guess}}$ ). Furthermore, the mixture model demonstrated that both of these reductions in $\mathrm{P}_{\text {Target }}$ (and increases in $\mathrm{P}_{\text {Guess }}$ ) were accompanied by an increase in $\mathrm{SD}$ (i.e. reduced precision). These results speak to the strength of applying a mixture modeling approach, as we were able to demonstrate that SD increased by over $25 \%$ from the simultaneous $\left(22.28^{\circ}\right)$ to the delayed mask offset conditions $\left(28.97^{\circ}\right.$ and $\left.28.43^{\circ}\right)$.

Surprisingly, $\mathrm{P}_{\text {Non-target }}$ was both non-zero and increased as a result of OSM. Whether this represents true location-feature misbinding, or whether when participants had no target memory, they adopted a strategy of responding with a non-masked item's orientation rather than guessing, is beyond the scope of this report. Importantly, less than $10 \%$ of trials were nontarget trials and excluding this component from the model (i.e., using a two component model like the one proposed by Zhang and Luck, 2008) did not change the main finding that delayed mask conditions in OSM produce degradation in the precision of a target's orientation.

That OSM can degrade a target's representation without it being 'substituted' or 'updated' out of conscious awareness seems to be inconsistent with object-mediated accounts of OSM and is perhaps more in line with the feedforward account proposed by Põder (2013). Põder's model shows that failures in target report in some OSM paradigms can 
a

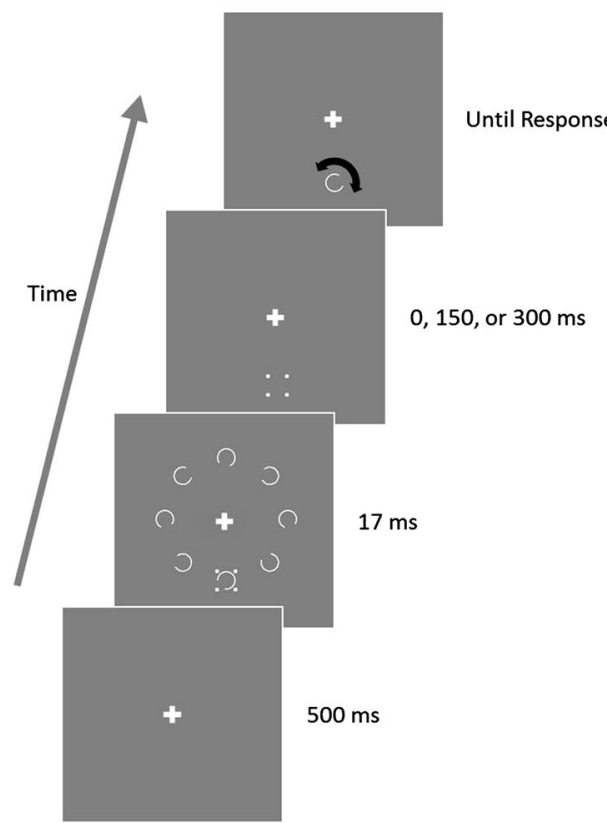

Fig. 1 Example trial sequences (set size eight) for Experiments 1 (a) and 2 (b). A fixation screen was followed by a brief search display which contained either two, four or eight Landolt-Cs. The target object is

be modeled by target degradation resulting from both signal decay and by the addition of mask noise to the target representation.

To test Põder's (2013) account Jannati, Spalek, and Di Lollo (2013) developed a novel OSM procedure that separated an initial "target plus mask" stimulus from a subsequent "mask only" stimulus. Because the contribution of noise by the mask

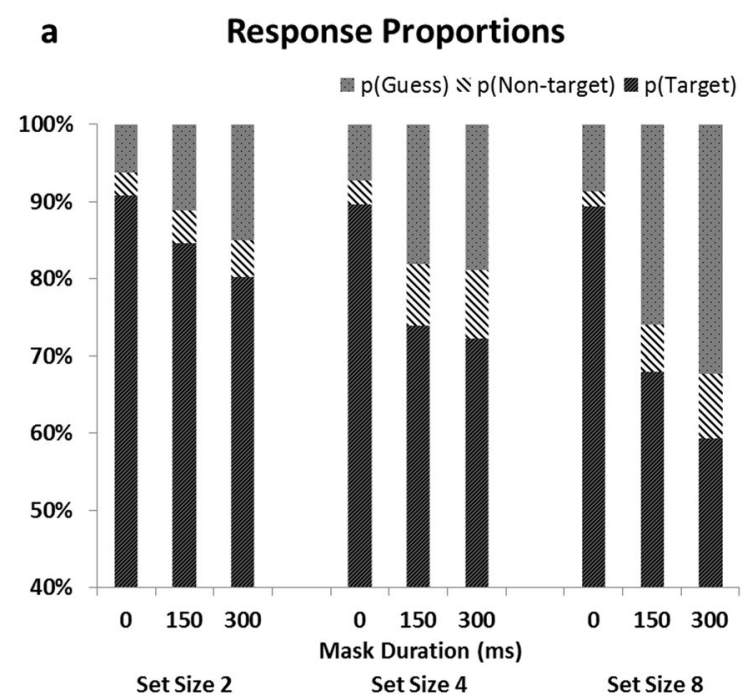

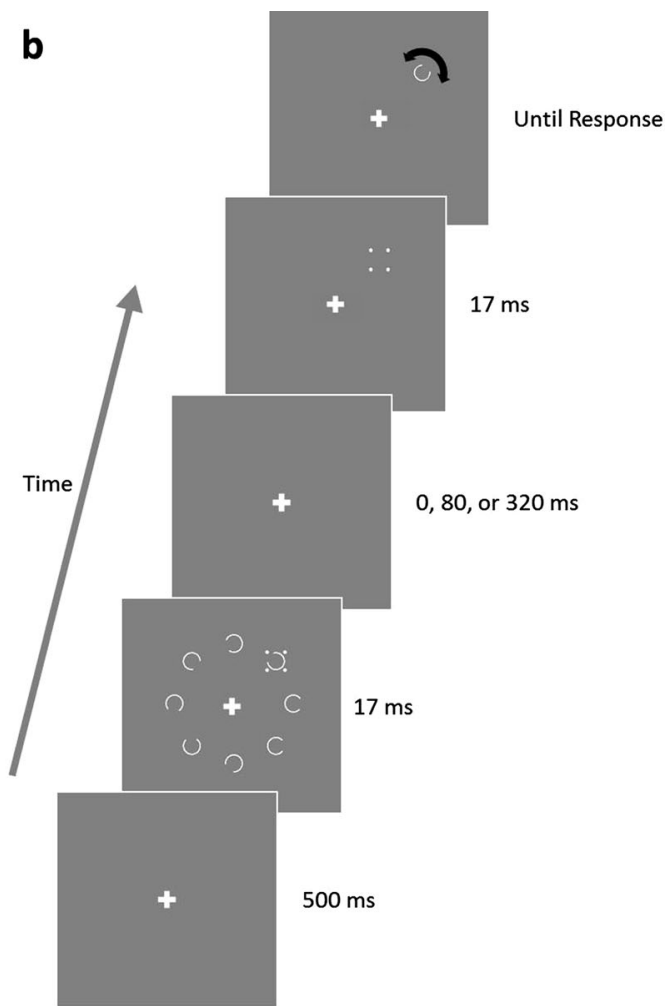

identified by the four-dot mask. Participants swiped fingers on a touchpad to recreate the target Landolt-C's gap orientation

is equated across conditions, and signal/target decay is assumed to decrease with increasing ISI, Põder's model predicts that OSM should increase with increasing ISI. However, Jannati et al. (2013) found that OSM peaked at an ISI of approximately $80 \mathrm{~ms}$ - an ISI close to the theorized time needed to execute communication between low- and high-level visual processes (Fahrenfort, Scholte, \& Lamme, 2007).

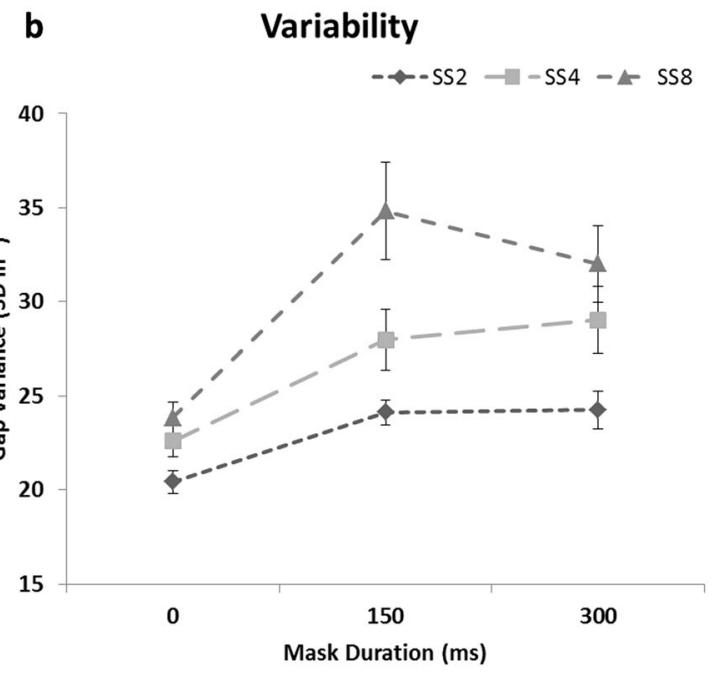

Fig. 2 Results for Experiment 1 of trial proportions for the three response types' parameter estimates (a) and mean variability (b). Error bars Standard errors of the mean 


\section{Experiment 2}

The purpose was to first replicate the OSM effect on $\mathrm{P}_{\text {Target }}$, $\mathrm{P}_{\text {Guess }}, \mathrm{P}_{\text {Non-target, }}$ and $\mathrm{SD}$ using the procedure developed by Jannati et al. (2013), and second to determine whether the vitiating effects of OSM on target precision observed in Experiment 1 would generalize to a paradigm designed to isolate object updating mechanisms from possible feedforward mechanisms.

\section{Method}

\section{Participants}

Twenty-three undergraduate students from Queen's University participated in exchange for credit in an introductory psychology course. All participants had normal or corrected-to-normal vision, and were naïve to the experiment's purpose.

\section{Apparatus, design, and procedure}

The apparatus, design and procedure were identical to Experiment 1 with the following exceptions (see Fig. 1b). We replaced the three mask durations with three ISI conditions $(0,80,320 \mathrm{~ms})$. ISI referred to the duration between the 'target and mask' offset and the mask-only onset, and were the same as those used in Experiment 3 of Jannatti et al. (2013). The duration of both the 'target and mask' display and mask-only display was fixed at $17 \mathrm{~ms}$.

\section{Results and discussion}

Data from one participant was excluded due to poor performance (accuracy in simultaneous mask offset conditions

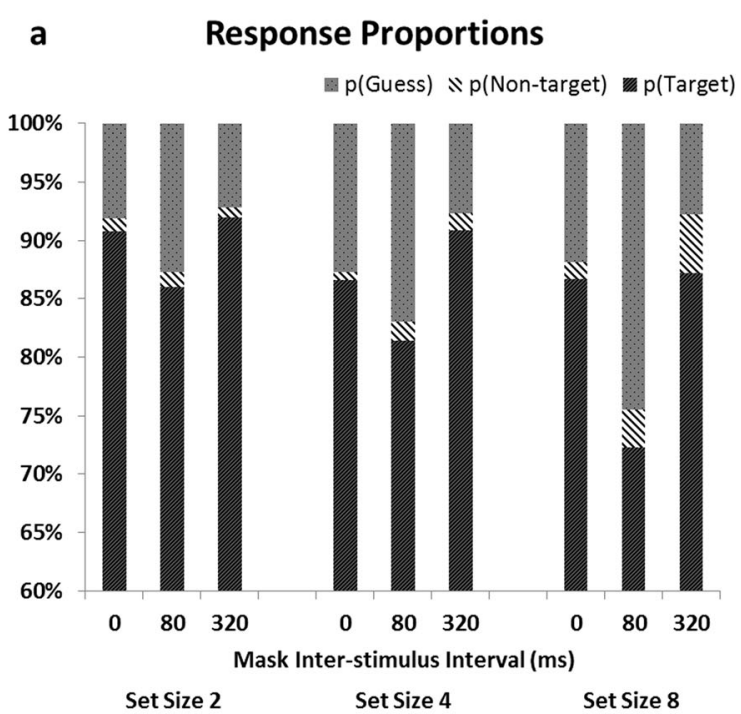

$<50 \%$ ). Four 3 (set size) $\times 3$ (mask ISI) ANOVAs were conducted —one for each parameter estimate $\left(\mathrm{P}_{\text {Target }}, \mathrm{P}_{\text {Non-target }}\right.$, $\mathrm{P}_{\text {Guess, }}$, and SD) derived from the mixture model (see Fig. 3). As with Experiment 1, the ANOVAs for both $\mathrm{P}_{\text {Target }}$ and $\mathrm{P}_{\text {Guess }}$ showed main effects of set size and ISI and an interaction, all $F_{\mathrm{S}}>4.94$, all $P_{\mathrm{S}}<.002$, all partial $\eta^{2}>.19$. To investigate the interaction, the magnitude of OSM for each set size was calculated as the difference between the critical $80 \mathrm{~ms}$ ISI and the average of the 0 and $320 \mathrm{~ms}$ ISI conditions. Paired samples $t$-tests revealed that OSM magnitude for $\mathrm{P}_{\text {Target }}$ did not differ between set sizes $2(5.3 \%)$ and $4(7.3 \%), t(21)=$ $1.02, P=.32$, but decreased from set size 4 to $8(14.6 \%)$, $t(21)=2.78, P=.011$. Similarly, OSM magnitude for $\mathrm{P}_{\text {Guess }}$ did not differ between set sizes $2(5.1 \%)$ and 4 $(6.7 \%), t(21)=0.89, P=.38$, but increased from set size 4 to $8(14.7 \%), t(21)=2.73, P=.012$.

Results for $\mathrm{P}_{\text {Non-target }}$ mirrored those of Experiment 1 in that there was a main effect of ISI, $F(2,42)=12.34$, $P<.001$, partial $\eta^{2}=.37$, such that there was no difference between the $0(1.1 \%)$ and $80 \mathrm{~ms}(1.3 \%)$ ISI conditions, $t(21)=0.38, P=.71$, but $\mathrm{P}_{\text {Non-target }}$ increased from the 80 to $320 \mathrm{~ms}$ ISI condition $(3.2 \%), t(21)=3.51, P=.002$. There was no effect of set size and no interaction, all $F_{\mathrm{S}}$ $<2.82$, all $P \mathrm{~s}>.07$, all partial $\eta^{2} \mathrm{~s}<.12$.

For SD (see Fig. 3b), there was a main effect of set size, $F(2,42)=8.26, P=.001$, partial $\eta^{2}=.28$, and mask duration, $F(2,42)=39.60, P<.001$, partial $\eta^{2}=.65$, but no interaction, $F(4,84)=0.65, P=.63$, partial $\eta^{2}=.03$. Contrast analyses revealed a linear increase in SD across set sizes, and a quadratic trend for ISI, all $P_{\mathrm{S}}<.001$, all partial $\eta^{2}>.50$. Critically, variance was greatest in the $80 \mathrm{~ms}$ ISI condition $\left(28.74^{\circ}\right)$ relative to the $0 \mathrm{~ms}\left(24.58^{\circ}\right)$ and $320 \mathrm{~ms}\left(23.14^{\circ}\right)$ conditions, all $P_{\mathrm{s}}<.001$.

As with Experiment 1, re-analysis with a two-component model did not alter the interpretation of the results. Crucially,

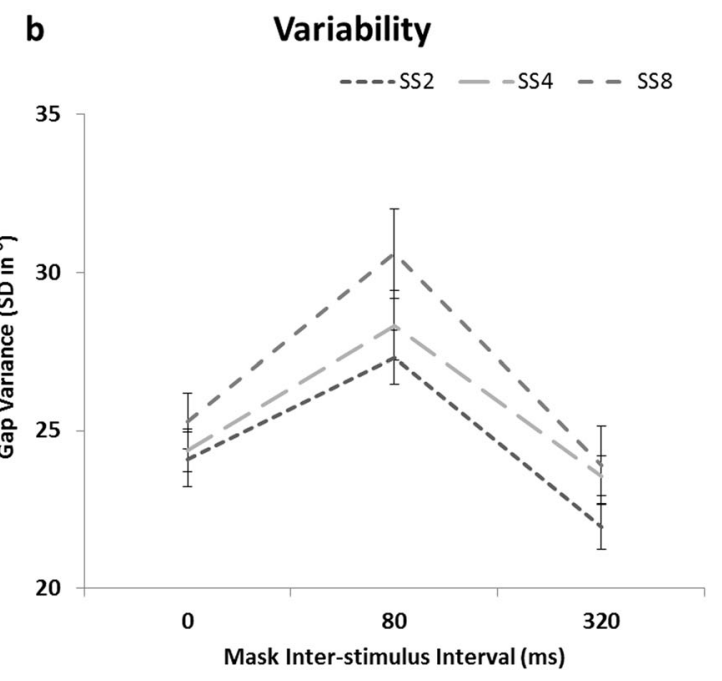

Fig. 3 Results for Experiment 2 of trial proportions for the three response types' parameter estimates (a) and mean variability (b). Error bars Standard errors of the mean 
these results corroborate the conclusion of Experiment 1 that object-updating mechanisms resulting from OSM cause perceptual degradation of target representations. Furthermore, by eliminating the role that a sustained mask may play in adding noise to a target's representation (as in the canonical OSM paradigm used in Experiment 1), Experiment 2 isolated an object-mediated process as cause of the vitiating effect.

\section{General discussion}

Standard forced-choice methodologies have limited our understanding of the effect of OSM on the quality of target representations. By using a continuous report measure and a mixture modelling approach, we demonstrated that canonical impairments in accuracy during OSM tasks are the result of both the target's elimination from consciousness as well as its deterioration within consciousness. We then replicated this result using a procedure that ruled out a feedforward account that could have accounted for the deterioration independent of object oriented processing. The two experiments converge on the conclusion that object-updating mechanisms degrade target representations within conscious awareness.

Our method of analysis revealed the now contentious interaction between set size and mask duration. Recently, Argyropoulos, Gellatly, Pilling, and Carter (2013) showed that the interaction - normally attributed to attention — could be eliminated by applying a guessing correction. Our findings were thus surprising as the mixture model estimates the independent contribution of guess responses and in essence then corrects for guesses. However, converging evidence suggests that ceiling level performance drives the interaction, something our design did not adequately account for (Filmer, Mattingley, \& Dux, 2014; Pilling, Gellatly, Argyropoulos, \& Skarratt, 2014). Importantly, it was not the purpose of this paper to address the existence of this contentious aspect of OSM but to characterize the effect of OSM on the quality of target representation.

Our results are consistent with previous results that showed an offset mask can cut off or "trim" a target without it being eliminated entirely from conscious (Kahan \& Enns, 2010). Our results extend this partial alteration of a conscious object by showing that object updating can also occur via a process of degradation. Notably, no theories of OSM (either object mediated or feedforward) in their current form describe how OSM might degrade the representation of a target. Thus, our findings call for elaboration of extant models of how object representations are modified when faced with discrepant perceptual input.

Given that precision decrements can result from inter-item competition (Ma, Husain, \& Bays, 2014), our finding that a mask can deteriorate a conscious object's representation may mean that, in the fight between target and mask for representation, OSM reflects mask prioritization due to its persistent perceptual input in the standard paradigm (Experiment 1), and its presentation during a hypothesized window for communication between early and late visual processing areas (Experiment 2). Either way, mask prioritization leads to reduced sampling of the target object representation and a consequent reduction in representational quality.

In conclusion, by applying a mixture model approach to two OSM paradigms, we have shown that the updating of object representations does not function in a discrete all-ornone fashion, but instead impacts the quality of object representations. This novel methodological approach and demonstration that object updating impacts the precision of object representations should provide new research avenues for the study of representation in conscious and unconscious vision.

Author notes This research was supported in part by the National Sciences and Engineering Research Council of Canada Award RGPIN341580-07 to D.E.W.

Ethical considerations All research was approved by the Queen's University graduate research ethics board and all participants provided informed consent before completing either experiment.

Conflict of interest The authors declare no conflict of interest with respect to the authorship or publication of this article.

Author contributions G.W.H. came up with the study concept. All authors contributed to the study design. Testing and data collection were performed by G.W.H. G.W.H. and J.R. performed the data analysis and interpretation under the supervision of D.E.W. G.W.H. drafted the manuscript, and J.R. and D.E.W. provided critical revisions. All authors approved the final version of the manuscript for submission.

\section{References}

Argyropoulos, I., Gellatly, A., Pilling, M., \& Carter, W. (2013). Set size and mask duration do not interact in object-substitution masking. Journal of Experimental Psychology. Human Perception and Performance, 39(3), 646-661.

Asplund, C. L., Fougnie, D., Zughni, S., Martin, J. W., \& Marois, R. (2014). The attentional blink reveals the probabilistic nature of discrete conscious perception. Psychological Science, 25(3), 824-831.

Bays, P., Catalao, R., \& Husain, M. (2009). The precision of visual working memory is set by allocation of a shared resource. Journal of Vision, 9, 1-11.

Brainard, D. H. (1997). The psychophysics toolbox. Spatial Vision, 10(4), 433-436.

Breitmeyer, B. G. (2007). Visual masking: Past accomplishments, present status, future developments. Advances in Cognitive Psychology, 3(1-2), 9-20.

Breitmeyer, B. G., \& Öğmen, H. (2006). Visual masking: Time slices through conscious and unconscious vision. Spatial Vision: Oxford University Press.

Dehaene, S., \& Naccache, L. (2001). Towards a cognitive neuroscience of consciousness: Basic evidence and a workspace framework. Cognition, 79(1-2), 1-37. 
Di Lollo, V., Enns, J. T., \& Rensink, R. A. (2000). Competition for consciousness among visual events: the psychophysics of reentrant visual processes. Journal of Experimental Psychology. General, 129(4), 481-507.

Enns, J. T. (2004). Object substitution and its relation to other forms of visual masking. Vision Research, 44(12), 1321-1331.

Fahrenfort, J. J., Scholte, H. S., \& Lamme, V. A. F. (2007). Masking disrupts reentrant processing in human visual cortex. Journal of Cognitive Neuroscience, 19(9), 1488-1497.

Filmer, H. L., Mattingley, J. B., \& Dux, P. E. (2014). Size (mostly) doesn't matter: The role of set size in object substitution masking. Attention, Perception, \& Psychophysics, 76(6), 1620-1629.

Giesbrecht, B., Bischof, W. F., \& Kingstone, A. (2003). Visual masking during the attentional blink: Tests of the object substitution hypothesis. Journal of Experimental Psychology. Human Perception and Performance, 29(1), 238-258.

Goodhew, S. C., Pratt, J., Dux, P. E., \& Ferber, S. (2013). Substituting objects from consciousness: A review of object substitution masking. Psychonomic Bulletin \& Review, 20(5), 859-877.

Jannati, A., Spalek, T. M., \& Di Lollo, V. (2013). A novel paradigm reveals the role of reentrant visual processes in object substitution masking. Attention, Perception, \& Psychophysics, 75(6), $1118-1127$.
Kahan, T. A., \& Enns, J. T. (2010). Object trimming: when masking dots alter rather than replace target representations. Journal of Experimental Psychology. Human Perception and Performance, 36(1), 88-102.

Lleras, A., \& Moore, C. M. (2003). When the target becomes the mask: Using apparent motion to isolate the object-level component of object substitution masking. Journal of Experimental Psychology. Human Perception and Performance, 29(1), 106-120.

Ma, W. J., Husain, M., \& Bays, P. M. (2014). Changing concepts of working memory. Nature Neuroscience, 17(3), 347-356.

Moore, C. M., \& Lleras, A. (2005). On the role of object representations in substitution masking. Journal of Experimental Psychology. Human Perception and Performance, 31(6), 1171-1180.

Pilling, M., Gellatly, A., Argyropoulos, Y., \& Skarratt, P. (2014). Exogenous spatial precuing reliably modulates object processing but not object substitution masking. Attention, Perception, \& Psychophysics, 76(6), 1560-1576.

Põder, E. (2013). Attentional gating models of object substitution masking. Journal of Experimental Psychology. General, 142(4), $1130-1141$.

Zhang, W., \& Luck, S. J. (2008). Discrete fixed-resolution representations in visual working memory. Nature, 453(7192), 233-235. 